\title{
Effects of partial quenching and staggered fermions on the scalar correlator
}

\author{
Sasa Prelovsek* \\ University of Ljubljana and Institute Jozef Stefan, Ljubljana, Slovenia \\ E-mail: sasa.prelovsek@ijs.si
}

\begin{abstract}
We determine the mass of the lightest $\bar{q} q$ scalar meson with $I=1$ using the simulation with two dynamical Domain Wall Fermions. The conventional exponential fit of the scalar correlator is justified in this case giving the mass $1.58 \pm 0.34 \mathrm{GeV}$. In general the scalar correlator receives also the bubble contribution, which is the intermediate state with two pseudoscalar mesons. This contribution is sizable at light quark masses and has to be incorporated in the fit of the scalar correlator in order to extract the scalar meson mass. We provide predictions for the bubble contribution in Partially Quenched ChPT, Staggered ChPT and ChPT for Mixed quark actions. We find that the bubble contribution is significantly affected by the unphysical approximations that are employed in simulations. It can render the negative sign and unphysical effective mass $2 M_{\pi}$ in the scalar correlator with $I=1$.
\end{abstract}

XXIIIrd International Symposium on Lattice Field Theory

25-30 July 2005

Trinity College, Dublin, Ireland

* Speaker. 


\section{Introduction}

The Nature of the lightest observed scalar resonances is not revealed yet. There are two experimentally well established scalar resonances $a_{0}(980)$ and $a_{0}(1450)$ with isospin $I=1$ below $2 \mathrm{GeV}$. It is still not clear which one of the two is the lightest $\bar{d} u$ scalar state. This raises a further question whether $a_{0}(980)$ is perhaps a tetraquark [1] if $a_{0}(1450)$ turns out to be the lightest $\bar{d} u$ scalar state.

The first issue could be settled with a determination of the lightest $\bar{d} u$ scalar mass on the lattice. For this purpose the lattice simulations evaluate the scalar correlator

$$
C(t)=\sum_{\vec{x}}\langle 0|\bar{d}(\vec{x}, t) u(\vec{x}, t) \bar{u}(\overrightarrow{0}, 0) d(\overrightarrow{0}, 0)| 0\rangle=A e^{-m_{a 0} t}+B(t)+\cdots .
$$

If $a_{0}$ is the lightest state with $I=1$ and $J^{P}=0^{+}$, the correlator (1.1) drops as $e^{-m_{a 0} t}$ at large $t$ and determination of $m_{a 0}$ is straight forward. Multi-hadron states with $J^{P}=0^{+}$and $I=1$ also propagate between the source and the sink and they often shadow the interesting part $e^{-m_{a 0} t}$ in the correlator (1.1). The most important multi-hadron state is the intermediate state with two pseudoscalars, we call it the bubble contribution $B(t)$ and display it in Fig. 1. In proper three flavor QCD the twopseudoscalar states are $\pi \eta, K \bar{K}, \pi \eta^{\prime}$, while $\pi \pi$ is not allowed by Bose symmetry and conservation of $J^{P}$ and $I^{G}$. In two flavor QCD the only state $\pi \eta^{\prime}$ is relatively heavy and therefore not so disturbing. The scalar correlator (1.1) is dominated by $B(t)$ in the simulations with light quark masses if $M_{P 1}+M_{P 2}<m_{a 0}$ which opens the decay channel $a_{0} \rightarrow P_{1} P_{2}$. So the bubble contribution has to be incorporated in the fit of the scalar correlator (1.1) in order to extract $m_{a 0}$.

In addition to the physical intermediate states $P_{1} P_{2}$, the bubble contribution incorporates also the effects of the unphysical approximations employed in the simulations. This allows the extraction of $m_{a 0}$ via (1.1) even in this case. The unphysical effects were first attributed to the bubble contribution in case of quenched QCD [2], where it describes the magnitude and the negative sign of the lattice correlator well [四, 邚.

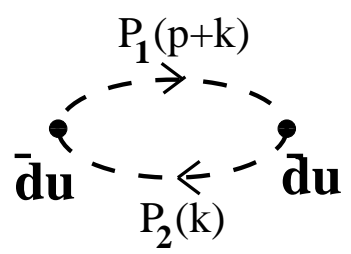

Figure 1: The bubble contribution to the scalar correlator. Here $P_{1}$ and $P_{2}$ denote pseudoscalar mesons in the relevant version of ChPT.

Here we present the analytical predictions for $B(t)$ in partially quenched simulations [ 4 ] and in simulations with staggered fermions or mixed-quark actions [5]. The results are obtained at the lowest order in the appropriate version of Chiral Perturbation Theory (ChPT) and apply for the point-point correlators.

We demonstrate the method of determining $m_{a 0}$ in a simulation with two dynamical Domain Wall Fermions (DWF) [凹]. The resulting scalar meson masses from the dynamical and partially quenched correlators agree.

The simulation with chiral (DWF) sea and valence quarks is presented in Section 2. Section 3 considers the simulations with staggered sea and valence quarks. The simulations with staggered sea and chiral valence are considered in Section 4. We conclude in Section 5. 


\section{Simulation with two dynamical Domain Wall quarks}

\section{Dynamical correlator with $m_{v a l}=m_{\text {sea }}$}

The simulation with two Domain Wall sea quarks and Domain Wall valence quarks [6] does not suffer from unphysical effects in contrast to the simulations that will be considered later. The conventional exponential fit $C(t)=A e^{-m_{a 0} t}$ (1.1) is justified in two-flavor QCD since the only intermediate state $\pi \eta^{\prime}$ is relatively heavy and $B(t)$ is small [ $\left[\right.$ ]. The resulting $m_{a 0}$ for three different quark masses is presented in Fig. 2a a, while the linear extrapolation to the chiral limit gives

$$
m_{a 0}^{d y n}=1.58 \pm 0.34 \mathrm{GeV} .
$$

This mass has large error-bar, but it is definitely above $1 \mathrm{GeV}$ and favors $a_{0}(1450)$ as the lightest $\bar{d} u$ scalar meson.

\section{(a) Dynamical $\quad$ (b) Partially Quenched}

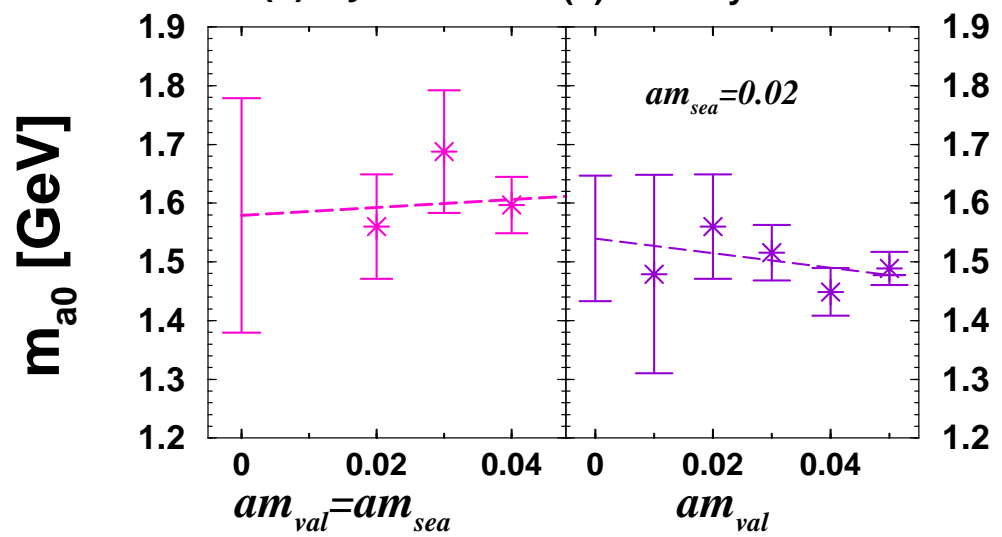

Figure 2: Mass of $a_{0}$ from a simulation with two dynamical Domain Wall quarks, $a^{-1} \simeq 1.7 \mathrm{GeV}$ and $V=16^{3} \times 32$ : (a) $m_{a 0}$ from dynamical scalar correlator with $a m_{v a l}=a m_{\text {sea }}$ using exponential fit; (b) $m_{a 0}$ from partially quenched scalar correlator with $a m_{\text {val }} \neq a m_{\text {sea }}=0.02$ using fit (1.1) with $B^{P Q C h P T}$ (2.2).

\section{Partially Quenched correlator with $m_{\text {val }} \neq m_{\text {sea }}$}

The partially quenched scalar correlator in Fig. 3 is positive for $m_{v a l}>m_{\text {sea }}$, whereas it is negative for $m_{v a l}<m_{\text {sea }}$ [凹]. The striking effect of partial quenching is attributed to the bubble diagram in Fig. 1. In order to compute the bubble diagram we need the coupling of the point scalar current to two pseudoscalars and the pseudoscalar propagators from Partially Quenched ChPT. The coupling is obtained from $\bar{d} u=-\partial \mathscr{L}^{C h P T} / \partial \mathscr{M}_{d u}$ and is equal to $B_{0}=M_{\pi}^{2} /\left(2 m_{q}\right)$ in any version of $C h P T$ at the lowest order. The bubble contribution for $m_{0} \rightarrow \infty$ is $B(t)=F . T$. $[B(p)]_{\vec{p}=0}$ with [ [

$$
B^{P Q C h P T}(p)=2 B_{0}^{2} \sum_{k}\left\{\frac{1}{(k+p)^{2}+M_{\text {val sea }}^{2}} \frac{1}{k^{2}+M_{\text {val }, \text { sea }}^{2}}-\frac{1}{(k+p)^{2}+M_{\text {val }, \text { val }}^{2}} \frac{k^{2}+M_{\text {sea }, \text { sea }}^{2}}{\left(k^{2}+M_{\text {val }, \text { val }}^{2}\right)^{2}}\right\} .
$$

It does not contain any free parameters, since pseudoscalar masses $M$ and $B_{0}=M_{\pi}^{2} /\left(2 m_{q}\right)$ are determined from the lattice data. The sum over the loop momenta $k$ is performed over the allowed discrete momenta on the lattice. Fig. 3 shows that the bubble contribution is positive for $m_{v a l}>$ $m_{\text {sea }}$ and negative for $m_{v a l}<m_{\text {sea }}$ like the lattice data. The negativity of the scalar correlator for 
$m_{v a l}<m_{\text {sea }}$ and large $t$ is described well by the bubble contribution. Having understood the source of the unphysical effect of partial quenching, we extract $m_{a 0}$ by fitting the partially quenched scalar correlator to (1.1) and display it in Fig. Da. The linear extrapolation to the chiral limit gives $m_{a 0}^{P Q}=1.51 \pm 0.19 \mathrm{GeV}$ in agreement with the dynamical result (2.1) and with smaller error.

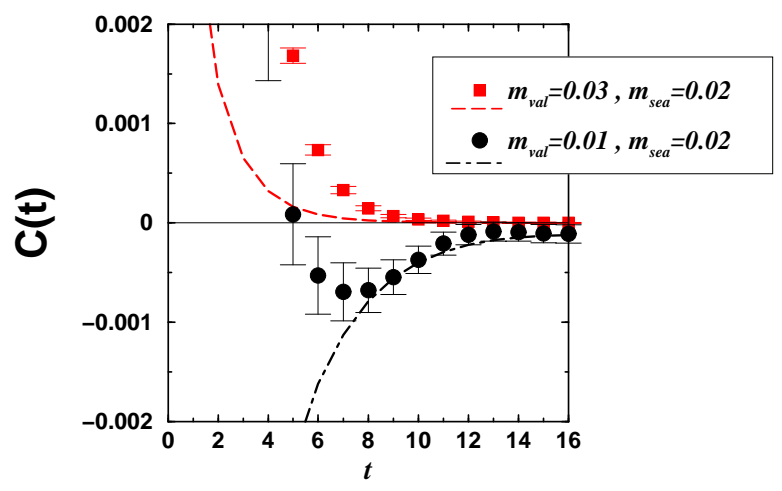

Figure 3: The symbols present the partially quenched point-point lattice correlators from a simulation with two dynamical Domain Wall quarks. The lines present the corresponding $B^{P Q C h P T}(t)(2.2)$.

\section{Simulations with staggered sea and valence quarks}

The scalar correlator was simulated using $2+1$ staggered sea quarks and staggered valence quarks by MILC [7] and UKQCD [8]. The authors of [7, 8] were surprised to find the effective mass significantly below $M_{\pi}+M_{\eta}$, although $\pi \eta$ is the lightest state with $I=1$ for light $u / d$ quarks in proper QCD.

This can be attributed to the taste breaking effects which enter the scalar correlator via the bubble contribution [5] in the Staggered ChPT of [9]

$$
\begin{aligned}
B^{S C h P T}(p)=B_{0}^{2} \sum_{k}\{- & 4\left[\frac{1}{(k+p)^{2}+M_{U_{I}}^{2}} \frac{1}{3} \frac{\left(k^{2}+M_{S_{I}}^{2}\right)}{\left(k^{2}+M_{U_{I}}^{2}\right)\left(k^{2}+\frac{1}{3} M_{U_{I}}^{2}+\frac{2}{3} M_{S_{I}}^{2}\right)}\right. \\
& \left.+\frac{1}{(k+p)^{2}+M_{U_{V}}^{2}} a^{2} \delta_{V} \frac{\left(k^{2}+M_{S_{V}}^{2}\right)}{\left(k^{2}+M_{U_{V}}^{2}\right)\left(k^{2}+M_{\eta_{V}}^{2}\right)\left(k^{2}+M_{\eta_{V}^{\prime}}^{2}\right)}+(V \rightarrow A)\right] \\
+ & \left.\frac{1}{16} \sum_{b=1}^{16}\left[2 \frac{1}{(k+p)^{2}+M_{U_{b}}^{2}} \frac{1}{k^{2}+M_{U_{b}}^{2}}+\frac{1}{(k+p)^{2}+M_{u s_{b}}^{2}} \frac{1}{k^{2}+M_{u s_{b}}^{2}}\right]\right\}
\end{aligned}
$$

with the notation from [9]. The forth-root trick is incorporated in (3.1) by weighting the diagrams with quark loops by factor $1 / 4$. The prediction has no free parameters since the pseudoscalar masses of various tastes [四] and hairpin parameters $a^{2} \delta_{V, A}^{\prime}$ [10] have been determined by MILC. In the continuum limit the pseudoscalars of various tastes are degenerate and $a^{2} \delta_{V, A}^{\prime} \rightarrow 0$, so the lightest intermediate state in (3.1) is $\pi \eta$. The taste breaking at finite $a$ makes possible the intermediate state with mass $2 M_{\pi}$ and is responsible that the effective mass of $B^{S C h P T}(t)$ is close to $2 M_{\pi}$, as shown in Fig. 6 . The effective mass of $B^{S C h P T}(t)$ qualitatively agrees with the effective mass obtained by MILC [7] and UKQCD [8] indicating that the lattice correlators are dominated by two-pseudoscalar intermediate states and are significantly affected by the taste breaking. 


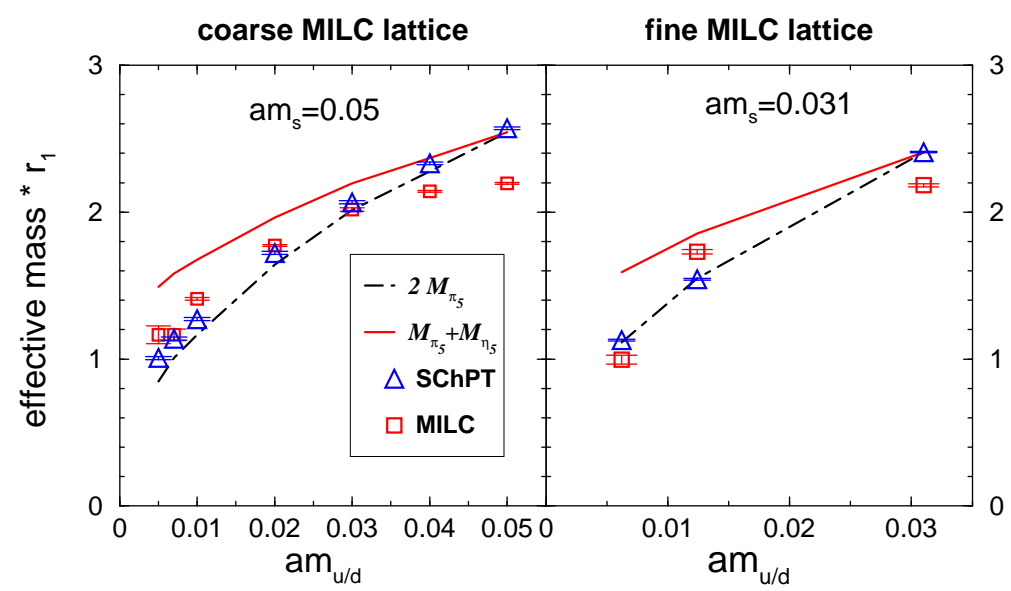

Figure 4: The squares represent the effective mass of the scalar correlator from MILC staggered simulation with $2+1$ dynamical quarks. The triangles present the effective mass of $B^{S C h P T}(t)(3.1)$ at $t=20$ for coarse MILC lattice and at $t=30$ for fine MILC lattice. Mass is plotted in units of $r_{1} \simeq 1.6 \mathrm{GeV}^{-1}$ like in 咘.

\section{Simulations with staggered sea quarks and chiral valence quarks}

The simulations with chiral valence quarks on the available staggered MILC configurations [11, 12] present an appealing possibility. However there is no unique recipe how to match valence and sea quark masses at finite $a$ and some features of partial quenching always remain.

The effect of mixed quark actions in simulations with $2+1$ staggered sea quarks can be attributed to the bubble contribution [5] within Mixed ChPT of [13] ${ }^{1}$

$$
\begin{aligned}
B^{M C h P T}(p)=B_{0}^{2} \sum_{k}\{ & -\frac{4}{3} \frac{1}{(k+p)^{2}+M_{v a l, v a l}^{2}} \frac{1}{\left(k^{2}+M_{v a l, v a l}^{2}\right)^{2}} \frac{\left(k^{2}+M_{U_{I}}^{2}\right)\left(k^{2}+M_{S_{I}}^{2}\right)}{k^{2}+\frac{1}{3} M_{U_{I}}^{2}+\frac{2}{3} M_{S_{I}}^{2}} \\
+ & \left.2 \frac{1}{(k+p)^{2}+M_{v a l, u}^{2}} \frac{1}{k^{2}+M_{v a l, u}^{2}}+\frac{1}{(k+p)^{2}+M_{v a l, s}^{2}} \frac{1}{k^{2}+M_{v a l, s}^{2}}\right\} .
\end{aligned}
$$

The only unknown parameter $a^{2} \Delta_{M i x} \equiv M_{v a l, s e a}^{2}-B_{0}\left(m_{\text {sea }}+m_{v a l}\right)$, sea $=u, s$, gives the taste breaking in the pion mass composed of one valence and one sea quark [13], while the other input parameters have been determined in [7, 11]. Fig. 5 shows that the scalar correlator can be negative for light $u / d$ quarks if valence and sea quark masses are tuned by matching $M_{v a l, v a l}=M_{\pi_{5}}$ (used by LHP [11]), while it is positive if $M_{v a l, v a l}=M_{\pi_{I}}$ are matched [5]. Comparison of the point-point lattice correlators and $B^{M C h P T}$ in case of matching $M_{v a l, v a l}=M_{\pi_{5}}$ offers a possibility to determine $a^{2} \Delta_{M i x}$.

\section{Conclusions}

We determined the mass of the lightest $\bar{q} q$ scalar meson with $I=1$ using the simulation with two dynamical Domain Wall Fermions. The exponential fit of the scalar correlator is justified in this case giving the mass $1.58 \pm 0.34 \mathrm{GeV}$. In general the scalar correlator receives also a sizable bubble contribution $B(t)$, which is the intermediate state with two pseudoscalar mesons. We provide analytical predictions for $B(t)$ for simulations that use (partial) quenching, staggered fermions

\footnotetext{
${ }^{1}$ Our result for $B^{M C h P T}(t)$ agrees in the limit $t \rightarrow \infty, a^{2} \Delta_{M i x} \rightarrow 0$ and $m_{u}=m_{d}=m_{s}$ with the result of [14], who study mixed quark actions but not the staggered sea.
} 

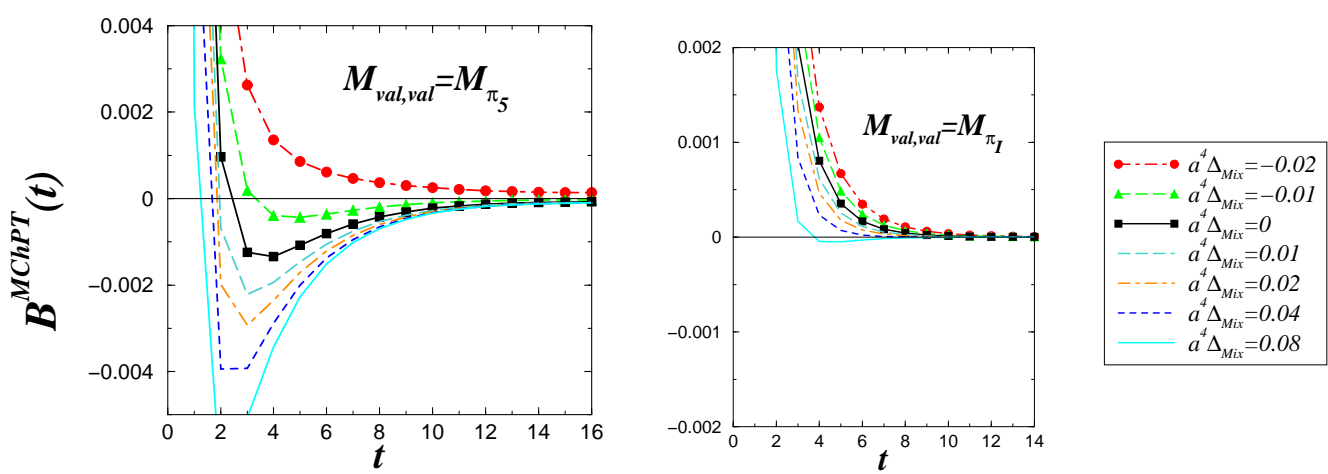

Figure 5: The bubble contribution $B^{M C h P T}(t)$ (4.1) for simulation with chiral fermions on coarse MILC configurations with sea quark masses $a m_{u / d}=0.01$ and $a m_{s}=0.05$. It is plotted for two choices of tuning $m_{v a l}$ with $m_{\text {sea }}$ and it depends on the value of $a^{2} \Delta_{M i x}$, which is varied in the reasonable range [5].

or mixed quark actions. Our predictions for $B(t)$ within relevant versions of ChPT are free of unknown parameters, expect for a parameter $a^{2} \Delta_{M i x}$ in case of mixed quark actions, which could be determined from the scalar correlator. We find that $B(t)$ is sizable at small quark masses and is significantly affected by the unphysical approximations that are employed in simulations. It can render the negative sign and unphysical effective mass $2 M_{\pi}$ in the scalar correlator. Our predictions for the bubble contribution will be needed in order to extract the scalar meson mass from the scalar correlator in the future simulations.

\section{References}

[1] M.G. Alford and R.L. Jaffe, Nucl. Phys. B 578 (2000) 367; N. Mathur, proceedings of Lattice05, POS(LAT2005)068; L. Maiani et al., Phys. Rev. Lett. 93 (2004) 212002.

[2] W. Bardeen et al. Phys. Rev. D 65 (2002) 014509 [hep-lat/ 0106008 ].

[3] S. Prelovsek and K. Orginos, Nucl. Phys. B (Proc. Suppl.) 119 (2003) 822 [hep-lat/0209132].

[4] S. Prelovsek et al., RBC Collaboration, Phys. Rev. D 70 (2004) 094503 [hep-lat/ 0407037 ].

[5] S. Prelovsek, Effects of staggered fermions and mixed actions on scalar correlator, to be published.

[6] Y. Aoki et al., RBC Collaboration, hep-lat/0411006

[7] C. Bernard et al., MILC Collaboration, Phys. Rev. D 64 (2001) 054506 hep-lat/ 0104002 ]; C. Aubin et al., MILC Collaboration, Phys. Rev. D 70 (2004) 094505 [hep-lat / 0402030 ].

[8] A. Irving et al., proceedings of Lattice05, POS(LAT2005)027.

[9] C. Aubin and C. Bernard, Phys. Rev. D 68 (2003) 034014 [hep-lat/ 0304014 ].

[10] C. Aubin et al., MILC Collaboration, Phys. Rev. D 70 (2004) 114501 [hep-lat / 0407028 ].

[11] D.B. Renner et al., LHP Coll., Nucl. Phys. Proc. Suppl. 140 (2005) 255 [hep-lat / 0409130 ].

[12] K.C. Bowler et al., UKQCD Collaboration, JHEP 0508 (2005) 003 [hep-lat/ 0411005 ].

[13] O. Bar, C. Bernard, G. Rupak and N. Shoresh, Phys.Rev. D72 (2005) 054502 [hep-lat/ 0503009 ].

[14] M. Golterman, T. Izubuchi and Y. Shamir, Phys. Rev. D 71 (2005) 114508 [hep-lat/ 0504013 ]. 